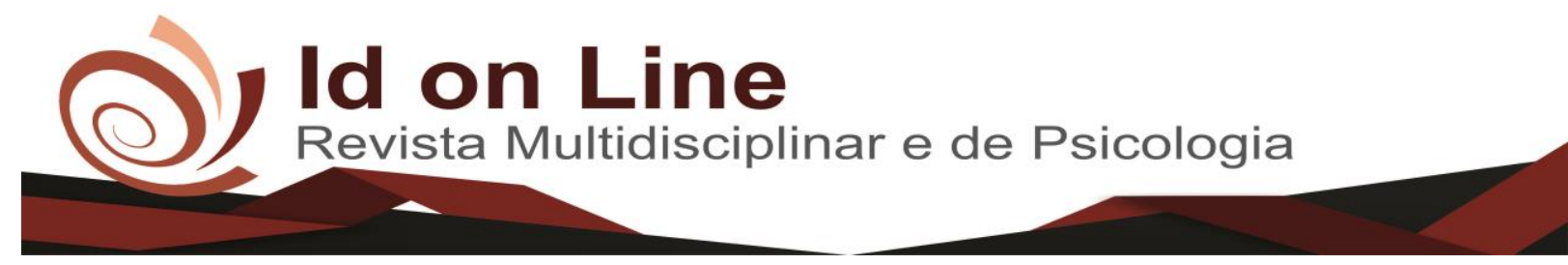

Artigo

\title{
Perfil Sócio-Econômico dos Consumidores de Medicamentos para Disfunção Erétil
}

\author{
Décio Santos Teixeira Júnior ${ }^{\text {; }}$ Stênio Fernando Duarte Pimentel ${ }^{2}$; Rafael Luiz Araújo Rodrigues ${ }^{2}$
}

\begin{abstract}
Resumo: A disfunção erétil (DE) tem como patologia predominantemente vascular originada por diversos fatores. Existem alguns medicamentos para essa patologia, contudo, grandes partes dos consumidores desses farmácos os utilizam sem terem a real necessidade simplismente para fim recreativo. Este trabalho objetivou traçar o perfil dos usuários desses medicamentos e verificar a ocorrência de uso recreativo dos mesmos. Pesquisa de carater transversal realizada atraves de aplicação de questionário a 410 homens em uma farmacia privada em um municipio do sudoeste Baiano. Dos entrevistados 52,2\% eram solteiros, 39,3\% tinham nível superior completo, $59,9 \%$ afirmaram que fariam ou já fizeram uso reacreativo desse medicamento e $21,2 \%$ fizeram uso mensal. Neste estudo observou-se que a maioria dos usuários de medicamentos para disfunção erétil não sofriam dessa patologia, utilizando-o com o intuito recreativo. A orientação do profissional farmacêutico é de suma importância na prevenção desta automedicação indevida, alertando sobre seus possíveis efeitos adversos que não são informados pela publicidade.
\end{abstract}

Palavras- Chave: Disfunção erétil. Uso recreativo. Inibidores da fosfodiesterase

\section{Socioeconomic Profile of Consumers of Medications for Erectile Dysfunction}

\begin{abstract}
Erectile dysfunction (ED) has a predominantly vascular pathology caused by several factors. There are some medicines for this pathology, however, large parts of the consumers of these pharmacies use them without having the real need simply for recreational purposes. This work aimed to trace the profile of the users of these drugs and to verify the occurrence of recreational use of them. Cross-sectional study carried out by means of questionnaire application to 410 men at a private pharmacy in a municipality in southwest Baiano. Of the interviewees, $52.2 \%$ were single, $39.3 \%$ had a complete upper level, 59.9\% said they would or would have used this drug, and $21.2 \%$ had a monthly use. In this study, it was observed that the majority of users of drugs for erectile dysfunction did not suffer from this pathology, using it for recreational purposes. The orientation of the pharmacist is of paramount importance in the prevention of this undue self-medication, alerting about its possible adverse effects that are not informed by the publicity.
\end{abstract}

Keywords: Erectile dysfunction. Recreational use Phosphodiesterase Inhibitors

\footnotetext{
${ }^{1}$ Graduando em Farmácia pela Faculdade Independente do Nordeste-FAINOR, Vitória da Conquista, Bahia, Brasil. Contato: deciojnr@yahoo.com.br;

${ }^{2}$ Doutor em Fisiologia e Fisiopatologia Clínica e Experimental. Docente na Faculdade Independente do NordesteFAINOR, Vitória da Conquista, Bahia, Brasil.

${ }^{3}$ Especialista em Perícias criminais e toxicológicas pela faculdade das águas emendadas, Planaltina - Distrito Federal. Docente na Faculdade Independente do Nordeste-FAINOR, Vitória da Conquista, Bahia, Brasil.
} 


\section{Introdução}

O desempenho sexual é um dos mais importantes aspectos da contribuição para o bem-estar psicológico do homem (BRITTO et al., 2010; BASTOS DE PAULA; DANIEL; BONFIM, 2012). A atividade sexual quando não se desenvolve por completo, é considerada como uma disfunção, que é resultante de uma perturbação em fases diferentes do ciclo de resposta sexual. Segundo o Manual Diagnóstico e Estatístico de Transtornos Mentais, uma disfunção sexual é caracterizada por perturbações nos processos que formam o esquema de resposta sexual, formado por Desejo, Excitação e Orgasmo (BRITTO et al., 2010; DSM 5, 2014).

A impotência pode ser uma patologia assustadora para o orgulho masculino e por este motivo é que, através dos séculos, muitas curas/soluções e tratamentos tenham sido propostos para o problema (AMARO; FONDA; COSTA, 2014). Muitos motivos induzem e promovem o uso irracional de medicamentos que aumentam a potência sexual, dentre esses estão curiosidade, diversão, estresse ou ainda um novo parceiro. Estudos comprovam que esses medicamentos não são indicados para pessoas que não sofrem de disfunção erétil (DE), uma vez que não há comprovação de que haja aumento da potência sexual nesses indivíduos (FERNANDES et al., 2012; ABDO; KAYATH, 2014; VILLEDA-SANDOVAL, 2012).

A disfunção erétil (DE) é uma patologia que acomete e tem causa principalmente vascular. Devido a isso, vários fatores podem ser considerados desencadeantes, entre eles diabetes, hipercolesterolemia, hipertensão e outros fatores como o envelhecimento, o sedentarismo e o fumo (BRITTO; BENETTI, 2010; FERNANDES et al., 2012).

O uso de iPDE 5 iniciou-se a partir de 1998, somente para pacientes com diagnostico de DE. Entretanto, há um número expressivo de homens que utilizam estes fármacos, mesmo não sofrendo DE. Entre essas pessoas, a maior quantidade é jovem que adquirem estes medicamentos em farmácias privadas e/ou pela internet sem a prescrição médica para buscar melhorar a função sexual (FERNANDES; RIBEIRO, 2012; VILLEDA-SANDOVAL et al., 2012; FREITAS et al., 2008).

Um importante iPDE 5 é o Citrato de Sildenafila, que foi veiculo de mudança sociocultural devido ao fácil uso e por não ter que realizar aplicações no pênis antes das relações, como era usado antigamente como terapia. Seu uso por pessoas não acometidas por DE, tornou-se uma prática comum e popular, devido à sua eficácia, segurança, comodidade e facilidade de administração por via oral, sendo esta atividade observada 
concomitantimente ao uso de drogas e álcool (AMARO et al., 2014; VILLEDASANDOVAL et al 2012).

O Sildenafila $\left(\right.$ Viagra $^{\circledR}$ ) foi o primeiro iPDE 5 disponível no mercado. Possui sua efetividade como inicio entre 30 e 60 minutos após sua administração, com resposta biológica durando ate 12 horas. Conduto uma alimentação muito gordurosa pode gerar uma redução ou retardar a sua absorção diminuindo seu tempo de ação (HORNUNG et al., 2012; FREITAS; CABIANCA, 2015). Os efeitos adversos principais encontrados incluem cefaleia, rubor facial e tontura, além de ter sido associada a algumas dificuldades visuais em menos de $2 \%$ dos pacientes. Porém, esses efeitos adversos são moderados e limitados ao uso contínuo (ABDO et al., 2002; BASTOS DE PAULA; DANIEL; BONFIM, (2012).

Nesse contexto, a grande necessidade e importância de profissionais ativos e comprometidos com a saúde dos pacientes, por exemplo, um farmacêutico ativo, de modo a desempenhar uma farmacoterapia correta e atenção farmacêutica, a fim de minimizar junto aos usuarios possíveis problemas relacionados aos medicamentos (PRM) e reações adversas aos mesmos (RAM).

Embora estes medicamentos sejam comercializados por meio de venda sob prescrição médica, existe certa facilidade em adquiri-los sem a prescrição devida. Diante de tal fato e do aumento do uso indiscriminado e sem orientação de medicamentos para DE hoje no mundo, o presente trabalho objetivou traçar o perfil dos usuários desses medicamentos e verificar a ocorrência de uso recreativo dos mesmos.

\section{Metodologia}

Pesquisa de caráter transversal, com abordagem quantitativa do tipo descritiva e exploratória, aprovada pelo Comitê de Ética e Pesquisa da Faculdade Independente do Nordeste em 23 de agosto de 2018 sob o parecer de número 2.234.738. Todos os pacientes do estudo assinaram o termo de consentimento referente à pesquisa.

\section{Participantes}

Foram realizadas entrevistas estruturadas com 410 homens clientes da farmácia privada na qual foi realizada o estudo, situada em um município no sudoeste baiano. Os critérios de 
inclusão foram: ser do sexo masculino e estarem na farmácia no momento da entrevista. Não foram incluídas mulheres e adolescentes do sexo masculino (menores que 18 anos) e os que não atendiam aos critérios de inclusão definidos previamente.

\section{Instrumento e procedimentos}

Foi aplicado um questionário elaborado conforme as necessidades do estudo pelos autores da pesquisa contendo questões relacionadas a se o paciente faz uso de medicamentos para disfunção erétil; em qual situação utiliza ou utilizaria o medicamento; e sobre conhecimento dos efeitos indesejáveis.

\section{Análise estatística}

Os dados foram digitalizados e tratados utilizando-se o programa Microsoft ${ }^{\circledR}$ Excel $^{\circledR}$ 2018, sendo considerada a frequência percentual as respostas dadas às questões presentes no instrumento de pesquisa utilizado.

\section{Resultados e Discussão}

A tabela 1 mostra os dados sociodemográficos dos participantes da pesquisa conforme sua frequência. Esta pesquisa também constatou que 52,2\% dos homens eram solteiros, 44,1\% declararam ser casados ou ter algum tipo de relacionamento estável e apenas 3,2\% eram divorciados. Apenas dois destes relatou ser viúvo. Com relação à renda mensal dos entrevistados, foi notado que a maioria recebe entre 3 a 5 salários $(37,3 \%)$.

Tabela 1. Dados sociodemográficos dos consumidores de medicamentos para disfunção erétil.

\begin{tabular}{llcc}
\hline Variáveis & & F & \% \\
\hline \multirow{3}{*}{ Estado civil } & Solteiro & 214 & 52,2 \\
& Casado & 181 & 44,1 \\
& Viúvo & 02 & 0,5 \\
& Divorciado & 13 & 3,2
\end{tabular}




\begin{tabular}{llcc} 
& De 3 a 5 salários mínimo & 84 & 20,5 \\
Renda familiar & De 5 a 8 salários mínimo & 168 & 41,1 \\
& Superior a 8 salário mínimo & 157 & 38,4 \\
& Ensino fundamental completo & 03 & 0,7 \\
Escolaridade & Ensino médio completo & 153 & 37,3 \\
& Ensino superior completo & 161 & 39,3 \\
& Ensino superior incompleto & 93 & 22,7 \\
\hline
\end{tabular}

F: frequência absoluta. Fonte: dados da pesquisa

Com relação à escolaridade, 37,3\% afirmaram possuir ensino médio completo e 39,3\% concluíram o ensino superior. Abdo e colaboradores (2006) também encontraram em seu levantamento que boa parte do público-alvo possuía ensino superior (56\%).

Já quando questionados sobre o uso do Sildenafila, 45,4\% dos entrevistados relataram ter utilizado em algum momento o Sildenafila. Contrário a esses dados, Hornung; Halila; Barbosa (2012) e DUARTE, 2010 constataram que 90\% da população estudada já haviam utilizado o Sildenafila. Acredita-se que muitas das respostas negativas relatadas no nosso estudo devem-se à vergonha de admitir o uso desse medicamento.

A tabela 2 mostra os dados referentes ao motivo do uso do medicamento, sendo que 59,9\% utilizaram-no recreativamente e 40,1\%, sob prescrição médica. Em seus estudos, Hornung; Halila; Barbosa (2012) e Freitas colaboradores (2008), observaram também essa maior proporção de respostas para o uso recreativo não prescrito da Sildenafila, com percentagem de $95 \%$ e $82 \%$ (70\% por curiosidade e $12 \%$ para potencializar a ereção), respectivamente.

Tabela 2. Motivos relatados pelos pacientes da pesquisa para uso do Sildenafila.

\begin{tabular}{llcc}
\hline Variáveis & F & $\%$ \\
\hline Situação que utilizou ou & Recreativo & 243 & 59,9 \\
utilizaria o medicamento & Prescrição médica & 163 & 40,1 \\
\hline
\end{tabular}

F: frequência absoluta. Fonte: dados da pesquisa

Dentre os pacientes que já utilizaram a Sildenafila, 21,2\% relataram o uso mensal do medicamento. Entretanto, nossa pesquisa não investigou se esse uso mensal foi decorrente 
de prescrição médica. Além disso, não foi investigado a frequência de utilização desses $40,1 \%$ que afirmaram fazer uso sob prescrição médica.

No presente trabalho, dos 59,9\% que confirmaram fazer uso recreativo de medicamento para DE adquiriram-no sem prescrição médica. Esses consumidores tais medicamentos sem ser acometido por DE, o que evidência um processo de automedicação e destaca uma possível prática ilegal de venda de medicamentos sem prescrição médica, o que é considerado um procedimento antiético. Como apresentado FREITAS et al., (2015) e HARTE; MESTON, 2011 este procedimento é praticado com frequência no Brasil, de forma que qualquer pessoa pode adquirir tais medicamentos.

Já em relação a possíveis reações adversas (RAM) decorrentes do uso de iPDE5, dos 45,4\% dos pesquisados que relataram fazer uso, 27,1\% afirmaram já haver manifestado algum tipo de RAM, e dentre as principais reações referidas foram taquicardia (batimentos cardíacos acelerados), cefaleia (dor de cabeça) e rubor facial. Estes resultados são semelhantes aos que foram apresentados por BARBOSA et al., (2012) e MOURA (2015).

Dados estes em conformidade com o estudo relatado por Barbosa et al. (2012), estes verificaram que dentre $14(38 \%)$ usuários de iPDE5, as principais reações adversas informadas foram dor de cabeça $5(37 \%)$ e rubor facial $3(22 \%)$. Também foram verificadas reações como suor exacerbado 1 (7\%), aceleração dos batimentos cardíacos 2 (11\%), ereção prolongada $1(6 \%)$ e hipertensão 1 (4\%). Os resultados também assemelham com os apontados por Freitas e col. (2008), que demostraram como principais reações adversas entre usuários de iPDE5: cefaleia (23\%) e rubor facial (10\%).

Portanto, uma educação contínua é de extrema e fundamental importância para promoção do uso racional e a correta utilização de preservativos e a necessidade de acompanhamento médico para o uso correto de iPDE5, que a figura do profissional farmacêutico na orientação se faz necessária, através da prática da assistência farmacêutica.

\section{Conclusões}

Através dos dados, verificou-se que a maior parte dos consumidores que adquiriram medicamentos para DE foi com o intuito recreativo ao invés de tratamento com prescrição médica. Apesar da ampla comercialização dessa categoria de medicamentos, ainda são raros os estudos que são propostos para verificar seu impacto na vida sexual e saúde dos usuários recreativos. Vale ressaltar a importância da orientacao do profissional farmacêutico 
sobre o uso de medicamentos para disfunção erétil, alertando sobre seus possíveis efeitos adversos, evitando assim complicações nesta automedicação. Além disso, o uso recreativo e indiscriminado pode aumentar as práticas sexuais de risco e, assim, a contaminação e disseminação de doenças sexualmente transmissíveis (DSTs).

\section{Referências}

ABDO, C. H. N.; KAYATH, M. J. Efeito do tratamento com sildenafila sobre qualidade da ereção e satisfação sexual de homens com disfunção erétil. Revista Brasileira de Medicina, p. 483-488, 2014.

ABDO, C. H. N.; OLIVEIRA JÚNIOR, W. M.; MOREIRA, E. D.; FITTIPALDI, J. A. S. Perfil sexual da população brasileira: Resultados do Estudo do Comportamento Sexual do Brasileiro (ECOS). Rev Bras Med. v. 59, n. 4, p. 250-7, 2002.

ALDRIDGE, J.; MEASHAM, F. Sildenafil (Viagra) is used as a recreational drug in England. Bmj, v. 318, n. 7184, p. 669-669, 1999.

ALONSO, R.; CHRISTIAN, V. S. I.; EDUARDO, G. C.; et al. Frecuencia del uso recreacional de inhibidores de fosfodiesterasa-5 y otros potenciadores para mejorar la función sexual. Rev Mex Urol, v. 72, n. 5, p. 250-255, 2012.

AMARO, A.; FONDA, C. A.; COSTA, L. A. M. R. Avaliação do consumo de medicamentos para disfunção erétil entre indivíduos do gênero masculino na região do Vale da Paraíba. 2014. 31 f. Monografia (Graduação em Farmácia) - Faculdade de Pindamonhangaba, Pindamonhangaba, 2014.

BASTOS DE PAULA, I. S. H.; DANIEL, J.; BONFIM, A. R. Disfunção erétil: da medicalização à integralidade do cuidado na Atenção Básica. Saúde do Homem no SUS, v. 1, p. 101-109, 2012.

BRITTO, R.; BENETTI, S. P. C. Ansiedade, depressão e característica de personalidade em homens com disfunção sexual Rev. SBPH, Rio de Janeiro, v. 13, n. 2, 2010.

DUARTE, D. V. Estudo sobre a prevalência do uso de medicamentos estimulantes de ereção em adultos de Pelotas. 2010. 96 f. Dissertação (Mestrado em Epidemiologia) Universidade Federal de Pelotas, Pelotas, 2010.

FERNANDES, W. S.; RIBEIRO, W. Uso Irracional Dos Inibidores Da Fosfodiesterase 5. XVII Encontro Latino Americano de Iniciação Científica, XIII Encontro Latino Americano de Pós-Graduação e III Encontro de Iniciação à Docência - Universidade do Vale do Paraíba, p. $1-5,2012$. 
FREITAS, A. V. R.; CABIANCA, L. O.; NATIVIDADE, R. V. P.; NABAS, J. M. A. B. B. Uso indiscriminado de citrato de sildenafila (Viagra ${ }^{\circledR}$ ). Conexão Eletrônica, v. 12, n. 1, 2015.

FREITAS, A. V. R; CABIANCA, L. O. Uso indiscriminado de Citrato de Sildenafila (Viagra) Rev. Conexão eletrônica - Três Lagoas, MS, v.12 n.12- 2015.

FREITAS, V. M.; MENEZES, F. G.; ANTONIALI, M. M. S; NASCIMENTO, J, W, L. Frequência de uso de inibidores de fosfodiesterase-5 por estudantes universitários. Rev Saúde Pública, v. 42, p. 965-967, 2008.

FREITAS, V. M.; MENEZES, F. G.; ANTONIALLI, M. M. S.; NASCIMENTO, J. W. L. Frequência de uso de inibidores de fosfodiesterase-5 por estudantes universitários. Revista de Saude Publica, v. 42, n. 5, p. 965-967, 2008.

HARTE, B. C.; MESTON, C. M. Recreational Use of Erectile Dysfunction Medications in Undergraduate Men in the United States: Characteristics and Associated Risk Factors. Arch Sex Behav, v. 40, n. 3, p. 597-606, 2011.

HORNUNG, M.; HALILA, G. C.; BARBOSA, V. Prevalência de universitários que fazem uso de medicamentos para disfunção erétil. Visão Acadêmica, v.13, n.2, p. 27-32, 2012. Manual diagnóstico e estatístico de transtornos mentais: DSM-5. Tradução: Maria Inês Corrêa Nascimento et al. - 5. ed. - Dados eletrônicos. - Porto Alegre: Artmed, 2014.

MOURA, L. R. Aspectos farmacológicos do citrato de sildenafila no tratamento da disfunção erétil. RBM. v. 5, p. 1-8, 2015.

VILLEDA-SANDOVAL Christian, GONZÁLEZ-CUENCA Eduardo, SOTOMAYOR-DEZAVALETA Mariano, FERIA-BERNAL Guillermo, CALAO-PÉREZ Manuel B., IBARRASAAVEDRA Raúl, QUIJADA-CARLTON Héctor, CASTILLEJOS-MOLINA Ricardo Alonso Frecuencia del uso recreacional de inhibidores de fosfodiesterasa-5 y otros potenciadores para mejorar la función sexual Rev. Mex Urol 2012;72(5): 250-255.

Como citar este artigo (Formato ABNT):

TEIXEIRA JÚNIOR, Décio Santos; PIMENTEL, Stênio Fernando Duarte; ARAÚJO, Rafael Luiz. Perfil Sócio-Econômico dos Consumidores de Medicamentos para Disfunção Erétil. Id on Line Rev.Mult. Psic., 2019, vol.13, n.43, p. 522-529. ISSN: 1981-1179.

Recebido: 29/11/2018;

Aceito: $03 / 12 / 2018$ 